\title{
Proteoglycans in Normal and Severely Osteoarthritic Human Cartilage
}

\author{
Nagaswamisri VASAN \\ Department of Anatomy, College of Medicine and Dentistry of New Jersey-New Jersey Medical School, \\ 100 Bergen Street, Newark, NJ 07103, U.S.A.
}

(Received 24 September 1979)

\begin{abstract}
Proteoglycans from osteoarthritic cartilage were compared with those from normal articular cartilage. Normal proteoglycan aggregates are larger in size and more homogeneous than those in osteoarthritis. Proteoglycan monomers from both sources gave two peaks on controlled pore glass-bead chromatography. Although the retarded material from normal cartilage showed an affinity for hyaluronate, the same material from osteoarthritic cartilage did not. The hyaluronate-binding capacity of the material which is partly in the void volume and partly retarded was similar in both types of cartilage. These results suggest that in osteoarthritic cartilage the proteoglycan aggregates are smaller and more heterogeneous and that the chondroitin sulphate side chains are shorter. They also indicate that there are two populations of proteoglycan, one with its hyaluronate-binding-protein region of core protein intact and the other either possessing an inactive binding region or totally lacking it.
\end{abstract}

Proteoglycans are one of the major components contributing to the elasticity of the articular cartilage (Harris et al., 1972; Scott, 1975). The chondroitin sulphate-rich region is probably essential for the resilience of the proteoglycans, because it contains a large number of negatively charged groups (Inerot et al., 1978). In osteoarthritis, the elasticity of articular cartilage is decreased (Kempson, 1974). Decreased proteoglycan content and disruption of collagen have also been reported in osteoarthritic cartilage (Brandt \& Palmoski, 1976; Muir, 1977; Bayliss \& Ali, 1978; Inerot et al., 1978). Studies on the nature of proteoglycans in severe osteoarthritis (in the present study) indicate that the proteoglycan molecules are small, capable of aggregation with hyaluronic acid to a variable extent, and have a smaller than normal polysaccharide-chain length.

\section{Materials and Methods}

\section{Tissues}

Human articular cartilage was obtained fresh from the operating room and used immediately or stored at $-70^{\circ} \mathrm{C}$ until use. Normal cartilage was obtained from patients (38-46 years old) who underwent amputations after multiple fractures of the femur, or were undergoing prosthetic replacement. Osteoarthritic cartilage was obtained from femoral heads of eight patients (age 58-65) and used for biomedical analysis. Care was taken to exclude osteophytic and fibrocartilage materials. Loss of metachromasia seen in section was used to grade the osteoarthritic conditions. Samples showing severe loss of metachromasia were pooled (i.e. three 58 years, two 60 years and three 65 years were the age groups pooled) and studied.

All reagents were analytical grade. Guanidinium chloride was ultrapure and $\mathrm{CsCl}$ was optical grade (Schwarz/Mann Biochemicals, Orangeburg, NY, U.S.A.). For column chromatography, Sepharose 2B was purchased from Pharmacia Fine Chemicals, Piscataway, NJ, U.S.A., and controlled pore glass beads (CPG-10-2500) were obtained from Electronucleonics, Fairfield, NJ, U.S.A.

\section{Analytical methods}

Uronic acid was determined by using glucuronolactone as standard by the method of Bitter \& Muir (1962). Hexosamines were measured by the method of Elson \& Morgan (1933) as modified by Antonopoulos et al. (1964). Papain digestion was done as described by Brandt et al. (1976).

\section{Extraction and isolation of proteoglycans}

Cartilage samples were cleaned, weighed and cut into small pieces (about $1 \mathrm{~mm}^{3}$ ). Non-aggregated proteoglycan was extracted with $10 \mathrm{vol}$. ( $\mathrm{v} / \mathrm{w})$ of $0.15 \mathrm{M}-\mathrm{NaCl}$, containing $0.10 \mathrm{M}-6$-aminohexanoic acid (Aldrich Chemical Co., Milwaukee, WI, U.S.A.), $0.005 \mathrm{M}$-benzamidine hydrochloride (Eastman Kodak, Rochester, NY, U.S.A.) and $10 \mathrm{~mm}-$ 
EDTA to inhibit proteolysis (Oegema et al., 1975). For other studies cartilage was directly extracted for $36 \mathrm{~h}$ at $40^{\circ} \mathrm{C}$ with $15 \mathrm{vol}$. of $4.0 \mathrm{M}$-guanidinium chloride/ $0.05 \mathrm{M}$-sodium acetate, $\mathrm{pH} 5.8$, containing the proteolytic inhibitors as mentioned above. The extracts were separated from the tissue by centrifuging in a Sorvall centrifuge at $12000 \mathrm{~g}$ for $30 \mathrm{~min}$ at $4^{\circ} \mathrm{C}$.

\section{Associative guanidinium chloride/CsCl-density- gradient centrifugation}

A portion of $4.0 \mathrm{M}$-guanidinium chloride extract was dialysed against $100 \mathrm{vol}$. of $0.5 \mathrm{M}$-guanidinium chloride/sodium acetate buffer, $\mathrm{pH} 5.8$, for $24 \mathrm{~h}$ at $4^{\circ} \mathrm{C}$. To the dialysis residue, solid $\mathrm{CsCl}$ was added to a density of $1.60 \mathrm{~g} / \mathrm{ml}$. Centrifugation was then performed in a Beckman model $L$ preparative ultracentrifuge with type 40 fixed-angle rotor at $15^{\circ} \mathrm{C}$ and $39000 \mathrm{rev} . / \mathrm{min}$ for $44 \mathrm{~h}$. The tube contents were collected as lower two-thirds (A1) and upper one-third (A2).

Dissociative guanidinium chloride/CsCl-densitygradient centrifugation of $A 1$ fractions

The A1 fraction was mixed with an equal volume of $7.5 \mathrm{M}$-guanidinium chloride. Solid $\mathrm{CsCl}$ was added to a density of $1.5 \mathrm{~g} / \mathrm{ml}$ and centrifugation was repeated. The centrifuge tube contents were divided into lower two-fifths (A1-D1), middle two-fifths (A1-D2) and upper one-fifth (A1-D3) fractions.

\section{Hyaluronate-binding study with various proteogly- can fractions}

Cocks-comb hyaluronic acid used in these experiments was kindly provided by Dr. E. A. Balazs, New York, U.S.A. A portion (1 mg) of the proteoglycan sample in $0.5 \mathrm{ml}$ of $0.5 \mathrm{M}$-guanidinium chloride was mixed with hyaluronate $(0.1 \mathrm{ml})$ containing $24 \mu \mathrm{g}$ of uronic acid. The mixture was left at room temperature for $60 \mathrm{~min}$ and was then dialysed against the elution buffer $(0.5 \mathrm{M}$-sodium acetate, $\mathrm{pH} 6.5)$ before being subjected to gel chromatography on a Sepharose $2 \mathrm{~B}$ column $(100 \mathrm{~cm} \times 0.9 \mathrm{~cm})$ at $4^{\circ} \mathrm{C}$. The proportion of proteoglycan bound to hyaluronate was determined from the amount of uronic acid eluted in the void-volume region of the column compared with the control proteoglycan chromatographed in the absence of hyaluronate (Harding ham \& Muir, 1974).

\section{Sucrose linear-density-gradient centrifugation of crude proteoglycan}

A sample of the $4.0 \mathrm{M}$-guanidinium chloride extract was used for this study. A linear sucrose gradient $(5-20 \%, w / v)$ containing $4.0 \mathrm{M}$-guanidinium chloride $/ 0.005 \mathrm{M}$-sodium acetate, $\mathrm{pH} 5.8$, was established (Vasan \& Lash, 1979). A portion of crude proteoglycan in $4.0 \mathrm{M}$-guanidinium chloride was layered over the sucrose gradient and centrifuged in a Beckman model L2-65B ultracentrifuge with a swinging-bucket rotor (SW 27.1) at $20^{\circ} \mathrm{C}$ for $28 \mathrm{~h}$ at $25000 \mathrm{rev} . / \mathrm{min}$. Tube contents were collected in $0.5 \mathrm{ml}$ fractions and uronic acid analysis was performed after dialysis.

Controlled pore glass-bead chromatography of proteoglycan fractions

A portion $(1 \mathrm{ml})$ of proteoglycan in $0.5 \mathrm{M}-\mathrm{NaCl}$ was applied to a CPG-10-2500 column $(100 \mathrm{~cm} \times 0.9 \mathrm{~cm})$ and eluted with $0.5 \mathrm{M}-\mathrm{NaCl}$ at a flow rate of $0.4 \mathrm{ml} / \mathrm{min}$ (Vasan \& Lash, 1978). Fractions $(1 \mathrm{ml})$ were collected and a portion of each was used for uronic acid determination. The remainder of the fractions comprising peak 1 and peak 2 were pooled and concentrated for further study. For hyaluronic acid-binding studies, the material from the peaks was subjected to dissociative guanidinium chloride/ $\mathrm{CsCl}$ centrifugation, with the lower twofifths of the tube contents (A1-D1) being used.

\section{Results}

Grossly, the arthritic cartilage showed a characteristic loss of glossiness, discoloration and erosion. Histological examination of arthritic cartilage sections showed significant loss of metachromatic-staining material in the extracellular material.

Extraction with 4.0 M-guanidinium chloride resulted in the release of about $59-60 \%$ (normal) and $72-80 \%$ (osteoarthritic) of uronic acid from the tissues. Low-density proteoglycans were present in both normal and osteoarthritic cartilage. In arthritic cartilage there is an increase in the proportion of these low-density proteoglycans (Table 1). The A2 fraction showed a $100 \%$ increase. On a dissociative gradient about $23 \%$ of the proteoglycan was present in the A1-D2 and A1-D3 fractions. The arthritic cartilage also showed a decrease in the content of uronic acid and hexosamine and in glucosamine/ galactosamine molar ratios (Table 2).

The osteoarthritic-cartilage proteoglycan (A1) was mostly in the included peak close to the void volume on Sepharose 2B chromatography, whereas normal A1 fraction gave a definite excluded peak and a broad included peak (Fig. 1). Furthermore, the A1 fraction from arthritic cartilage showed a second distinct peak. All disaggregated (A1-D1) fractions were included in the gel and were polydisperse (Fig. 2). In normal cartilage the A1-D1 fraction contained an additional very small amount of excluded proteoglycan, which was not present in the osteoarthritic specimen. Sucrose-density-gradient centrifugation (Fig. 3) of crude $4.0 \mathrm{M}$-guanidinium chloride extract of proteoglycan from arthritic cartilage separated under dissociative conditions (5-20\% sucrose containing $4.0 \mathrm{M}$-guanidinium chloride) 
Table 1. Distribution of cartilage proteoglycans after associative and dissociative guanidinium chloride/CsCl-densitygradient centrifugation

Proteoglycans were extracted by using $4.0 \mathrm{M}$-guanidinium chloride and centrifuged as described in the Materials and Methods section.

\begin{tabular}{|c|c|c|c|}
\hline \multirow[b]{2}{*}{ Fractions } & \multirow[b]{2}{*}{ Cartilage } & \multicolumn{2}{|c|}{ Uronic acid (\% of total) } \\
\hline & & Normal & Arthritic \\
\hline Al (lower two-thirds) & & 82.0 & 71.0 \\
\hline A1-D1 (lower two-fifths) & & 75.1 & 48.0 \\
\hline A1-D2 (middle two-fifths) & & 5.7 & 18.7 \\
\hline A1-D3 (upper one-fifth) & & 1.2 & 4.3 \\
\hline A2 (upper one-third) & & 18.0 & 29.0 \\
\hline
\end{tabular}

Table 2. Percentage composition of proteoglycans

The chemical analysis was performed on the proteoglycan extracted with $4.0 \mathrm{M}$-guanidinium chloride from the cartilage, as described in the Materials and Methods section.

\begin{tabular}{|c|c|c|c|}
\hline & Cartilage & Normal & Arthritic \\
\hline $\begin{array}{l}\text { Uronic acid } \\
\text { Hexosamine }\end{array}$ & & $\begin{array}{l}24.9 \\
29.8\end{array}$ & $\begin{array}{l}18.0 \\
27.7\end{array}$ \\
\hline $\begin{array}{l}\text { Glucosamine/galactosamine } \\
\text { molar ratio }\end{array}$ & & $1: 1.1$ & $1: 1.9$ \\
\hline
\end{tabular}

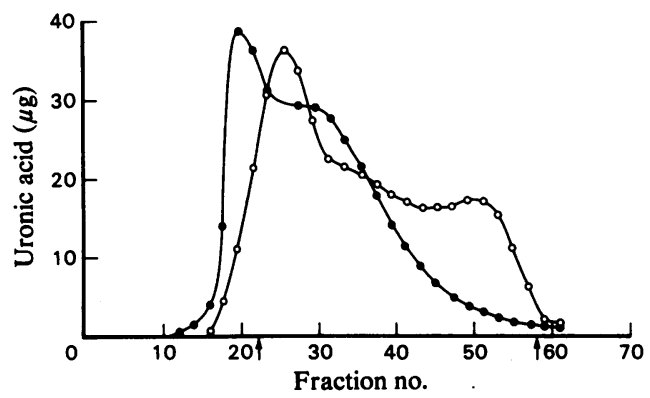

Fig. 1. Sepharose $2 B$ column $(100 \mathrm{~cm} \times 0.9 \mathrm{~cm})$ chromatograms of fraction Al from the associative-densitygradient centrifugation

The proteoglycan was dialysed against the elution buffer $(0.5 \mathrm{M}$-sodium acetate, $\mathrm{pH} 6.5)$ before application on the column, and the eluent collected in $1 \mathrm{ml}$ fractions. The void volume (fraction 22) and total volume (fraction 58) are marked by the arrows.

, Normal cartilage; $\mathrm{O}$, arthritic cartilage.

revealed three distinct populations of molecules. This is in contrast with the two populations present in normal cartilage. Proteoglycans from arthritic cartilage are of lower density than those of normal cartilage.

Arthritic cartilage when subjected to $0.15 \mathrm{M}-\mathrm{NaCl}$ extraction yielded more non-aggregated proteoglycan than did normal cartilage. Gel chromatography on Sepharose 2B (Fig. 4) showed that all nonaggregated proteoglycan was included by this gel. In the present study, gel chromatography of nonaggregated proteoglycan (Fig. 4) showed that there is no size difference between the normal and

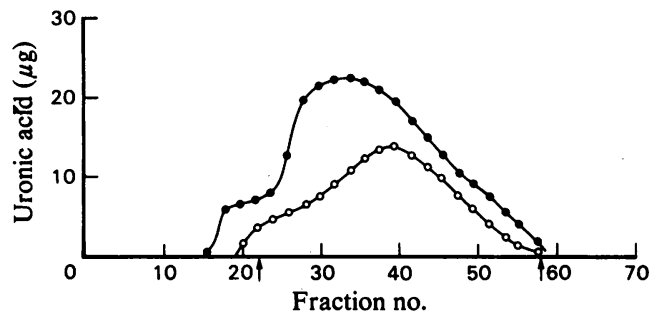

Fig. 2. Sepharose $2 B$ column $(100 \mathrm{~cm} \times 0.9 \mathrm{~cm})$ chromatograms of fraction A1-D1 from the dissociative-density-gradient centrifugation

The proteoglycan was dialysed against the elution buffer ( $0.5 \mathrm{M}$-sodium acetate, $\mathrm{pH}$ 6.5) before application on the column, and the eluent collected in $1 \mathrm{ml}$ fractions. The void volume (fraction 22) and total volume (fraction 58) are marked by the arrows. $\bullet$, Normal cartilage; $O$, arthritic cartilage.

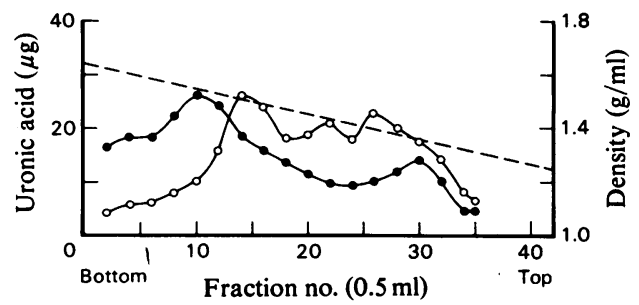

Fig. 3. Sucrose-density-gradient centrifugation profile of proteoglycan extracted from cartilage

A linear sucrose gradient $(5-20 \%, w / v ;----)$ containing $4.0 \mathrm{M}$-guanidinium chloride was established. The conditions for centrifugation are as described in the Materials and Methods section. $\bullet$, Normal cartilage; $O$, arthritic cartilage. 


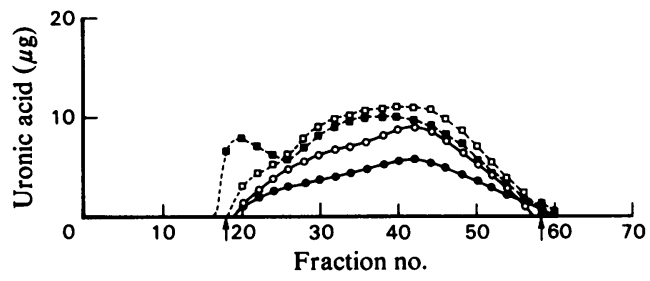

Fig. 4. Sepharose $2 B$ column $(100 \mathrm{~cm} \times 0.9 \mathrm{~cm})$ chromatogram of non-aggregate fraction in the absence and presence of $1 \%(w / w)$ hyaluronate

Iso-osmotic-saline extracts of cartilage were chromatographed alone (, normal; $O$, arthritic) or in presence of hyaluronate ( $\square$, normal; $\square$, arthritic). Samples containing about $1.0 \mathrm{mg}$ of uronic acid were applied to a column $(100 \mathrm{~cm} \times 0.9 \mathrm{~cm})$ of Sepharose 2B, eluted with $0.5 \mathrm{M}$-sodium acetate buffer, $\mathrm{pH} 6.5$, and collected in fractions of $1 \mathrm{ml}$. The void volume (fraction 18) and total volume (fraction 58) are shown by arrows.

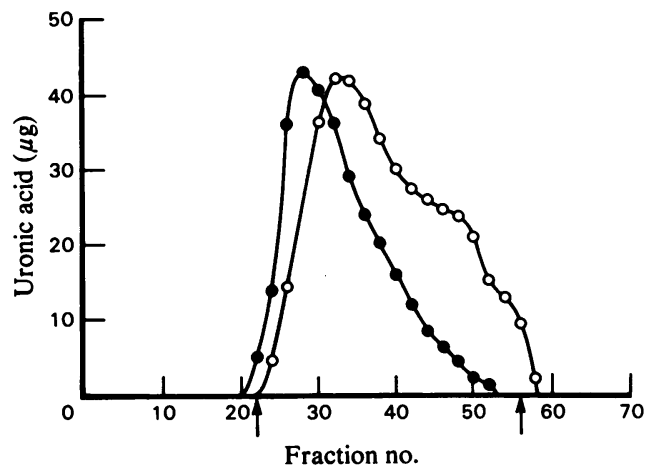

Fig. 5. Sephadex $G-200$ column $(60 \mathrm{~cm} \times 1.0 \mathrm{~cm}) \mathrm{chrom}$ atograms of the papain-digest fraction $A 1$ from the associative density-gradient centrifugation

The column was eluted by using $0.4 \mathrm{M}$-sodium acetate buffer, $\mathrm{pH} 6.8$, and $1 \mathrm{ml}$ fractions were collected. The patterns represent the chondroitin sulphate side chains. - Normal cartilage; $O$, arthritic cartilage. The void volume (fraction 22) and total volume (fraction 58) are shown by arrows.

arthritic-cartilage samples. Incubation of the nonaggregated proteoglycan with cocks-comb hyaluronic acid showed that the arthritic-cartilage proteoglycans were incapable of binding (Fig. 4). In contrast, a small but definite proportion, approx. $8-10 \%$ of non-aggregated proteoglycan from normal cartilage, was capable of interaction with hyaluronic acid. On the basis of Sephadex G-200

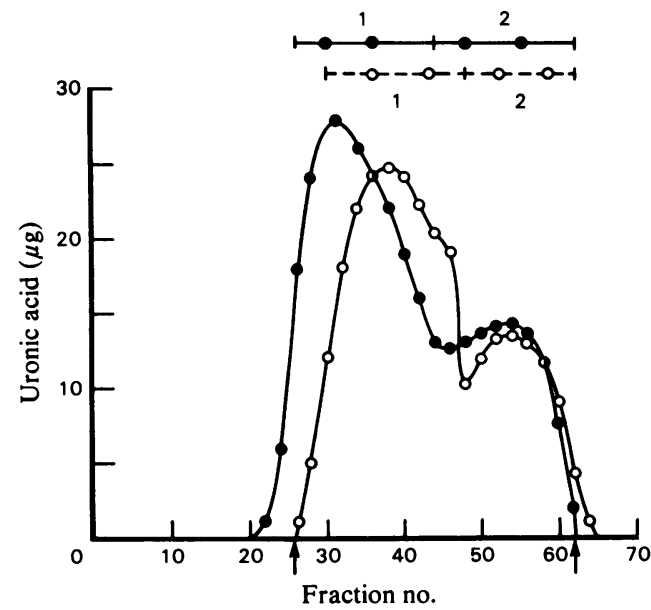

Fig. 6. Controlled pore glass-bead (CPG-2500) column chromatogram $(100 \mathrm{~cm} \times 0.9 \mathrm{~cm})$ of the Al-DI fraction from the dissociative-density-gradient centrifugation Proteoglycan fraction was dialysed against the elution buffer $(0.5 \mathrm{M}-\mathrm{NaCl}, \mathrm{pH} 6.5)$ before application on the column. The elution resulted in a profile which contained peak 1 and peak 2 ., , Normal cartilage; $O$, arthritic cartilage. The void volume (fraction 2) and total volume (Fraction 62) are shown by arrows.

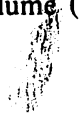

chromatography of papain-digested A1 fraction, the chondroitin sulphate chain of arthritic-cartilage A1 fraction is shorter than that of normal cartilage, as well as being more heterogeneous (Fig. 5). Crude proteoglycan extracted from normal and arthritic cartilage was subjected to controlled pore glass-bead chromatography (Fig. 6). Although both samples gave two distinct peaks (designated as peaks 1 and 2 ), the proteoglycan peaks from arthritic cartilage were retarded on the same column.

Peak-1 and peak-2 fractions were pooled and a portion was treated with papain and applied on a Sephadex G-200 column (Fig. 7). Peak 1 from arthritic cartilage showed the chondroitin sulphate side chain to be slightly smaller than normal (Fig. $7 a$ ). The peak-2 elution pattern showed a definite decrease in size and a greater degree of heterogeneity (Fig. $7 b$ ).

The ability of material from peaks 1 and 2 obtained from normal and arthritic cartilage to interact with cocks-comb hyaluronic acid was also determined. The pooled fractions from peaks 1 and 2 were subjected to dissociative guanidinium chloride/ $\mathrm{CsCl}$ centrifugation, and the lower two-fifths containing disaggregated molecule was examined. Peak1 material from both the samples showed a similar hyaluronic acid-binding capacity, but peak-2 

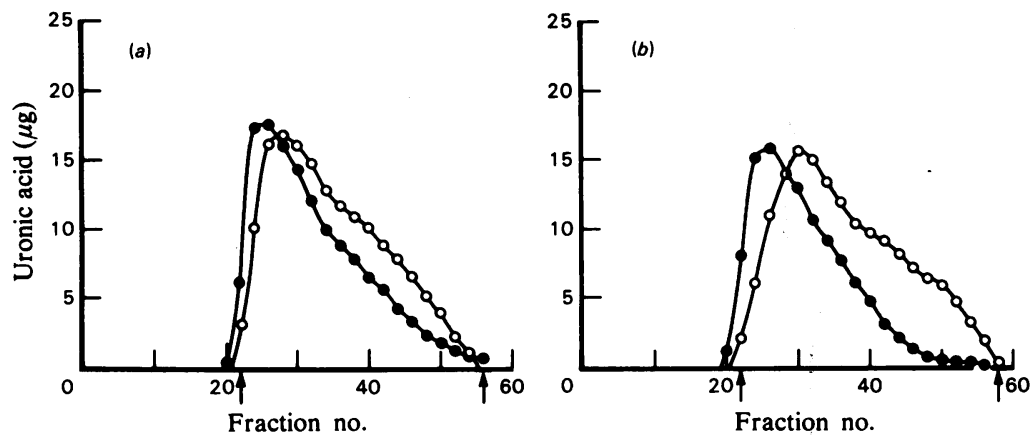

Fig. 7. Elution patterns of material from (a) peak 1 and (b) peak 2 (Fig. 6) after digestion with papain Peaks 1 and 2 were obtained by eluting A1-D1 fractions on a CPG-2500 column (see Fig. 6). Material from these peaks was subjected to papain digestion and chondroitin sulphate side-chain length was determined. This Figure depicts the elution profile on a Sephadex G-200 column $(60 \mathrm{~cm} \times 1.0 \mathrm{~cm})$ of the digest. 0 , Normal cartilage; 0 , arthritic cartilage. The void volume (fraction 22 ) and total volume (fraction 58 ) are shown by arrows.

Table 3. Special features of A1-D1 fractions

\begin{tabular}{|c|c|c|}
\hline & Cartilage & Normal \\
\hline $\begin{array}{l}\text { Size of proteoglycan monomer } \\
\text { Ability to bind with hyaluronate }\end{array}$ & & $\begin{array}{l}\text { Normal } \\
\text { Normal }\end{array}$ \\
\hline Size of glycosaminoglycan side chain & & Normal \\
\hline
\end{tabular}

* Peak 2 of proteoglycan monomer on controlled pore glass-beads column (Fig. 6) showed inability to bind with hyaluronate, whereas peak 1 could bind (Fig. 8), showing the presence of two types of proteoglycan population with different binding capacity.

† Shown by papain digestion of (1) A1-D1 fractions (Fig. 5) and (2) peaks-1 and -2 (Fig. 6) material isolated on a controlled pore glass-beads column (Figs. $7 a$ and $7 b$ ).

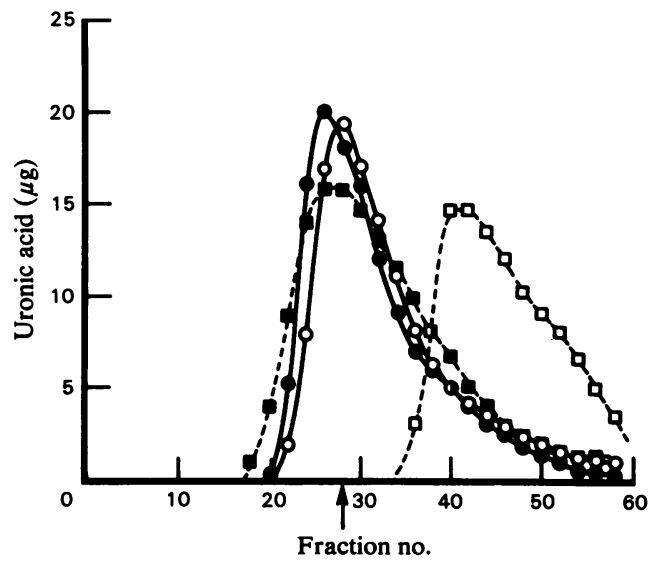

Fig. 8. Controlled pore glass-bead (CPG-2500) column $(100 \mathrm{~cm} \times 0.9 \mathrm{~cm})$ chromatogram of material from peak 1 and peak 2 (obtained as shown in Fig. 6) in the presence of cocks-comb hyaluronate

Peak 1: 0 , Normal cartilage; $O$, arthritic cartilage. Peak 2: $\square$, Normal cartilage + hyaluronate; $\square$, arthritic cartilage + hyaluronate. For details see the text. The arrow indicates the void volume (fraction 28). material of arthritic cartilage failed to display a normal binding (Fig. 8).

\section{Discussion}

The proteoglycans of cartilage are heterogeneous and vary in size and composition. Proteoglycans from osteoarthritic cartilage, however, showed a higher degree of heterogeneity (Table 3), containing more of the low-density components than normal (Table 1). The composition of osteoarthritic cartilage shown in Table 2 is consistent with earlier reports (Inerot et al., 1978; Bayliss \& Ali, 1978; Brandt, 1974). The finding of a higher galactosamine/glucosamine ratio in osteoarthritic human cartilage is in agreement with a previous report on dogs (McDevitt et al., 1977).

When proteoglycan from both normal and osteoarthritic cartilage was subjected to Sepharose 2B chromatography the normal proteoglycan gave an excluded and a retarded peak, whereas osteoarthritic-cartilage proteoglycan was mostly retarded, with a small amount in the void volume. When the 
proteoglycans from these cartilage samples were subjected to sucrose-density-gradient centrifugation under dissociative conditions (Fig. 3), the normal proteoglycan gave a typical bimodal pattern. In contrast, osteoarthritic-cartilage proteoglycan contained three distinct populations, which were of lower density than normal. Although the results of gel chromatography and sucrose-gradient centrifugation confirm the findings of others (Brandt \& Palmoski, 1976; Bayliss \& Ali, 1978; Brandt, 1974) that proteoglycans in osteoarthritis are smaller, the present paper indicates for the first time that the proteoglycan populations are more heterogeneous as well. In view of the fact that osteoarthritis is a condition of old age, it is possible that the decrease in the aggregated proteoglycan could be due to normal wear and tear, constant friction at the joint which is less lubricated by synovial fluid. When normal cartilage is subjected to considerable shear force by mechanical agitation or homogenization, the extracted proteoglycans are more heterogeneous and smaller than those obtained when the cartilage is subjected to milder extraction methods (Sajdera \& Hascall, 1969; N. Vasan, unpublished work).

Iso-osmotic-saline extraction of osteoarthritic cartilage yields increased amounts of non-aggregated proteolycans compared with normal articular cartilage. Proteoglycan aggregates are not dissociated by iso-osmotic salt solutions, which extract predominantly non-aggregated proteoglycans from normal cartilage. The core-protein amino acid composition of the non-aggregated proteoglycan is also different from that of disaggregated proteoglycans (Hardingham \& Muir, 1974). This suggests, therefore, that the proportion of aggregated proteoglycan in the osteoarthritic cartilage had declined, so that the resulting non-aggregated proteoglycan became extractable by solutions of low molarity. Non-aggregated proteoglycan extracted from osteoarthritic cartilage with sodium acetate buffer has also been shown to be unable to bind with hyaluronic acid (Brandt \& Palmoski, 1976; Brandt et al., 1976).

In the present study a successful attempt was made to isolate different populations of proteoglycan by means of chromatography on a controlled pore glass-bead column. Two peaks were isolated from both normal and osteoarthritic-cartilage proteoglycans. Peak-1 proteoglycan from osteoarthritic cartilage showed a considerable binding capacity to cocks-comb hyaluronic acid, whereas peak-2 material failed to show such affinity. This indicates that in osteoarthritis there are two populations of proteoglycans with respect to hyaluronic acid-binding capacity, one with the hyaluronic acid-binding region of the core protein intact and the other without it. The loss of this hyaluronic acid-binding region in osteoarthritis could be due to either enzymic degradation or mechanical factors (to a very limited extent; $\mathbf{N}$. Vasan, unpublished work), as mentioned above. It may also reflect an inactive binding region. This conclusion is supported by the finding of a neutral proteinase in osteoarthritic cartilage, which is capable of degrading proteoglycans (Sapolsky et al., 1974). In an earlier paper (Brandt, 1974) it was shown that the osteoarthritic-cartilage proteoglycans are capable of aggregation with hyaluronic acid, and that diminished aggregation of proteoglycans may be due to an abnormality in some other constituent of the aggregates. The results of the present study are in agreement with the observation by Brandt (1974) in that the chondroitin sulphate side chain of osteoarthritic-cartilage proteoglycans is smaller than normal, and that this is true for both peaks obtained on a controlled pore glass-bead column. In contrast with the observations of Brandt et al. (1976), however, the present study shows a decrease in hyaluronate-binding-region protein in the osteoarthritic-cartilage proteoglycan monomer. There is also evidence that the protein cores of proteoglycans from arthritic cartilage have been degraded, since the hydrodynamic size of dissociated proteoglycans from arthritic cartilage is decreased compared with that of normal cartilage (Fig. 3, sucrose-densitygradient centrifugation under dissociative condition). Thus it is possible that the chemical characteristics of the arthritic cartilage vary depending on the severity of the disease condition.

I am grateful to Dr. Leon Sokoloff and Dr. Elizabeth Alger for the critical evaluation and helpful suggestions in the preparation of this manuscript. This investigation was supported by a grant from National Institute of Health Biomedical Research Support 5 S07 RR05393 to the College of Medicine and Dentistry of New Jersey-New Jersey Medical School.

\section{References}

Antonopoulos, C. A., Gardell, S., Sziramai, J. A. \& De Tyssonk, E. (1964) Biochim. Biophys. Acta 83, 1-9

Bayliss, M. T. \& Ali, S. Y. (1978) Biochem. J. 169, 123-132

Bitter, T. \& Muir, H. (1962) Anal. Biochem. 4, 330-334

Brandt, K. D. (1974) Biochem. J. 143, 475-478

Brandt, K. D. \& Palmoski, M. (1976) Arthritis Rheum. 19, 209-215

Brandt, K. D., Palmoski, M. J. \& Perricone, E. (1976) Arthritis Rheum. 19, 1308-1314

Elson, L. A. \& Morgan, W. T. J. (1933) Biochem. J. 27, 1824-1828

Hardingham, T. E. \& Muir, H. (1974) Biochem. J. 139, 565-581

Harris, E. D., Jr., Parker, H. G., Radin, E. L. \& Krane, S. M. (1972) Arthritis Rheum. 15, 497-503

Inerot, S., Heinegard, D., Audell, L. \& Olsson, S. E. (1978) Biochem. J. 169, 143-156 
Kempson, G. E. (1974) in Dynamics of Connective Tissue Macromolecules (Burleigh, P. M. C. \& Poole, A. R., eds.), pp. 277-308, North-Holland Publishing Co., Amsterdam

McDevitt, C. A., Gilbertson, E. \& Muir, H. (1977) J. Bone Jt. Surg., Br. Vol. 59, 24-35

Muir, H. (1977) Ann. Rheum. Dis. 36, 198-199

Oegema, T. R., Jr., Hascall, V. C. \& Dziewiatkowski, D. D. (1975) J. Biol. Chem. 250, 6151-6159
Sajdera, S. W. \& Hascall, V. C. (1969) J. Biol. Chem. 244, 77-87

Sapolsky, A. I., Howell, D. S. \& Woessner, J. R., Jr. (1974) J. Clin. Invest. 53, 1044-1055

Scott, J. E. (1975) Philos. Trans. R. Soc. London, Ser. B 271, 235-242

Vasan, N. S. \& Lash, J. W. (1978) Connect. Tissue Res. 6, 191-199

Vasan, N. S. \& Lash, J. W. (1979) J. Embryol. Exp. Morphol. 49, 47-59 